\title{
Estudo de cenários para o gerenciamento dos resíduos sólidos urbanos de Curitiba
}

\section{Scenario studies for the management of urban solid waste in Curitiba}

\author{
Lucas Araújo de Melo \\ Mestre em Gestão Ambiental pela Universidade Positivo (UP). Engenheiro de Bioprocessos e Biotecnologista pela Universidade Federal do Paraná (UFPR) \\ Klaus Dieter Sautter \\ Doutor em Ciências Florestais pela UFPR. Agrônomo pela UFPR. Professor da Disciplina de Gerenciamento de Resíduos da UP

\section{Paulo Roberto Janissek} \\ Doutor em Química Orgânica pela Universidade de São Paulo (USP). Químico pela Universidade Federal de Santa Maria (UFSM). Professor da Disciplina de \\ Gerenciamento de Resíduos da UP
}

\section{Resumo}

Na cidade de Curitiba, a discussão relacionada aos resíduos sólidos tem se ampliado nos meios popular, acadêmico e político devido às dificuldades de se encontrarem formas de tratamento e destinação que possam substituir a atual disposição no aterro sanitário da Caximba. A técnica de cenários tem sido utilizada em diversas áreas como ferramenta do planejamento estratégico, e foi empregada neste artigo com o objetivo de auxiliar o planejamento da gestão de resíduos sólidos urbanos (RSU) em Curitiba. São apresentados dados sobre geração, caracterização e gerenciamento atual dos RSU, bem como dados socioeconômicos de seus habitantes. A caracterização dos resíduos sólidos urbanos em Curitiba indica uma geração de $0,6 \mathrm{~kg} \cdot \mathrm{hab}^{-1}$. dia-1 ${ }^{-1}$ valor considerado baixo em relação à média Brasileira. No entanto, a composição de resíduos recicláveis é de $40 \%$ do total destinado ao aterro sanitário. Uma metodologia para a simulação de cenários futuros da produção de RSU em Curitiba foi desenvolvida, e alguns exemplos de prováveis cenários foram construídos e comparados com o intuito de demonstrar uma aplicação da técnica, assim como de contribuir para o gerenciamento dos resíduos sólidos de Curitiba e para o desenvolvimento de estratégias e políticas de gestão. Dentre os cenários simulados para o período de 2008-2020, destaca-se que, para uma redução de $20 \%$ do material reciclável, uma economia de $\mathrm{R} \$ 2.410 .000,00$ e uma redução de 535.00 ton de resíduos sólidos podem ser alcançadas em relação ao cenário atual ("do nothing").

Palavras-chave: gerenciamento de resíduos sólidos; técnica de cenários; planejamento urbano.

\section{Abstract}

In Curitiba, the discussion on solid waste has increased at the popular, academic and political spheres due to the difficulties on finding ways of treatment and disposals to replace the current waste disposition at the Caximba sanitary landfill. The scenarios technique has been used in many areas as a tool for strategic planning. This article brings a characterization of municipal household waste production and management in Curitiba, and presents the scenario technique as an approach to waste management planning. The study indicates that $0,6 \mathrm{~kg}$.for inhabitant are daily generated, a value that is below the Brazilian cities average. Despite this low rate, the amount of recyclable material was found to be $40 \%$ of the total amount destined to the landfill. A methodology for the building of future scenarios of household waste production in Curitiba was developed, and some hypothetical scenarios were built and compared in order to demonstrate the application of the technique and to contribute with municipal solid waste management in Curitiba, and with the development of strategies and management policies. Among the simulated scenarios for the period of 2008-2020, stands out that for a $20 \%$ reduction of recyclable material, economical savings of about $\mathrm{R} \$ 2.410 .000$ and reduction of 535.000 ton. of solid waste may be achieved in comparison to the current scenario ("do nothing").

Keywords: solid waste management; scenario techniques, municipal planning.

Endereço para correspondência: Klaus Dieter Sautter - Rua Professor Pedro Viriato Parigot de Souza, 5.300 - 81280-330 - Curitiba (PR), Brasil - e-mail: ksautter@up.edu.br. Recebido: 08/05/09 - Aceito: 04/11/09 - Reg. ABES: 05409 


\section{Introdução}

Uma das grandes preocupações da humanidade em termos ambientais é a crescente geração de resíduos sólidos urbanos (RSU), consequência do aumento populacional, da rápida industrialização e do crescimento do consumo (AMORIN, 1996), além disso contribuem para a crescente geração de resíduos o modelo de desenvolvimento e do padrão de consumo e estilo de vida contemporâneo disseminado pelo capital. Em uma cidade, o planejamento da gestão de resíduos sólidos é fundamental, e nesse contexto, a utilização de dados históricos pode contribuir para a compreensão do seu processo de geração. Para isso, é importante que haja uma base histórica confiável e mecanismos para coleta de dados acerca da geração e composição dos resíduos sólidos gerados pela população. Principalmente em países em desenvolvimento, há uma falta de dados históricos sobre a produção de resíduos, o que dificulta o planejamento (DYSON; CHANG, 2005).

A técnica de cenários é uma ferramenta que tem sido utilizada no planejamento estratégico em diversas áreas e pode ser útil na gestão de resíduos sólidos em cidades, países e empresas. A possibilidade de se projetarem cenários futuros da geração de resíduos sólidos urbanos pode contribuir para o planejamento e desenvolvimento de estratégias ótimas de gerenciamento (DASKALOPOULOS; BADR; PROBERT, 1998). Vários autores utilizam a técnica de cenários para projetar a geração de resíduos sólidos e sua influência no futuro. Sokka, Antikainen e Kauppi (2007) utilizaram dados sobre os RSU e o crescimento demográfico observado para projetar cenários até o ano 2020. De modo similar, Daskalopoulos, Badr e Probert (1998) e Adhikari, Barringt e Martinez (2006) utilizaram tendências históricas para construir cenários futuros da geração de RSU em cidades e países. A técnica de cenários consiste em um método disciplinado para se identificarem possíveis futuros como parte do processo de planejamento estratégico. Segundo Buarque (2003), para a construção dos cenários, parte-se de um modelo mental (teórico) que interpreta as variáveis centrais e as interações entre elas, reduzindo-se a complexidade da realidade. Um aspecto muito importante desta técnica é a coleta de informações, etapa que subsidia a formulação dos princípios teóricos de interpretação do sistema em análise (CARIDADE; WRIGHT; SPERS, 2006).

A técnica de cenários, aplicada ao gerenciamento dos RSU na cidade de Curitiba, é particularmente importante levando-se em consideração o esgotamento iminente da capacidade do único aterro sanitário de Curitiba - o Aterro da Caximba. Existe, portanto, a necessidade de se discutirem as possibilidades e estratégias de gestão de resíduos sólidos. Assim, estudos técnicos sobre o problema dos resíduos sólidos na cidade podem contribuir para a melhoria da gestão destes resíduos, ajudando a direcionar e otimizar as ações e recursos aplicados.

Nesse contexto, o objetivo do presente trabalho foi discutir a questão dos RSU em Curitiba e sugerir a técnica de cenários futuros como ferramenta para o planejamento da gestão. A partir de dados socioeconômicos e da produção de resíduos sólidos urbanos, foi caracterizado o cenário atual. Desenvolveu-se e aplicou-se um método prospectivo para a construção de cenários alternativos da produção destes resíduos na cidade. O trabalho destaca também a importância do planejamento a longo prazo, que pode ser realizado com base em dados históricos

\section{Metodologia}

\section{Local de estudo}

O presente estudo de caso foi realizado para a cidade de Curitiba, capital do Estado do Paraná, situada a $25^{\circ} 25^{\prime}$ de latitude sul e $49^{\circ} 16$ de longitude oeste, 934,6 $\mathrm{m}$ acima do nível do mar, no primeiro planalto paranaense. É uma das maiores e principais cidades da região Sul do Brasil (IPPUC, 2007).

\section{Caracterização do cenário atual dos RSU em Curitiba}

Foram coletadas informações junto aos gestores públicos dos resíduos sólidos urbanos de Curitiba sob a forma de entrevista semiestruturada. A caracterização da produção de RSU na cidade foi feita através da análise do histórico de dados relativos à geração e à composição baseado no estudo de caracterização gravimétrica realizado por Tavares (2007). Dados do gerenciamento dos resíduos sólidos urbanos de 1990 a 2007, como quantidades geradas e custos com transporte e disposição, foram obtidos nos documentos fornecidos pelo Departamento de Limpeza Urbana de Curitiba (PAES et al, 2007).

\section{Cenários futuros da produção de RSU em Curitiba}

Para a construção dos cenários futuros, considerou-se que os fatores críticos, ou seja, os principais parâmetros que podem influenciar a produção de resíduos sólidos urbanos em uma cidade, em termos de quantidade e composição, são 'população' e 'padrão de vida', conforme adotado por Daskalopoulos, Badr e Probert (1998) e Sokka, Antikainen e Kauppi (2007).

A projeção dos cenários da produção de RSU em Curitiba foi baseada na associação dos fatores críticos com a produção de resíduos sólidos, a partir da tendência histórica desses parâmetros. Os fatores utilizados para obter e descrever as tendências históricas foram:

\section{Produção de RSU em Curitiba}

Os dados históricos da produção de RSU em Curitiba foram obtidos diretamente com o Departamento de Limpeza Pública da Secretaria do Meio Ambiente de Curitiba (Paes et al, 2007). 


\section{População de Curitiba}

A tendência histórica da população de Curitiba foi analisada a partir do histórico do crescimento populacional. Os dados históricos foram publicados pelo Instituto de Pesquisa Planejamento Urbano de Curitiba (IPPUC), e são provenientes de contagens populacionais, censos demográficos e estimativas do Instituto Brasileiro de Geografia e Estatística (IBGE) e do Instituto Paranaense de Desenvolvimento Econômico e Social (IPARDES) (IPPUC, 2007).

\section{PIB de Curitiba}

O produto interno bruto (PIB) per capita para a cidade de Curitiba foi obtido da base de dados - BDE web - do IPARDES (2002 a 2005) e em publicações do IPPUC (1995 a 2000). (IPPUC, 2007; IPARDES, 2008). O valor do PIB de 2001 para Curitiba não estava disponível nas publicações.

A utilização do PIB como variável preditiva foi feita de acordo com algumas considerações. Neste trabalho, assumiu-se que o PIB indicaria a capacidade da população de adquirir bens e produtos. Diante disso, para cumprir os objetivos da análise, foi necessário ajustar os valores, pois da forma como foram disponibilizados nos bancos de dados consultados, não medem o real poder de compra dos consumidores, pois não consideram a variação de preços dos produtos ao longo dos anos, ou seja, a inflação, ou o aumento do custo de vida para a população.

Para ajustar os valores de PIB a preços constantes e obter valores mais significativos para a análise, utilizou-se um índice de custo de vida que sintetiza a variação dos preços para todos os produtos consumidos pela população. Dentre os índices disponíbilizados pelo IBGE, foi utilizado o IPCA (Índice Nacional de Preços ao Consumidor Amplo), que mede a variação dos custos e dos gastos da população com alimentos, bebidas, produtos residenciais, habitação, saúde, despesas pessoais, transporte, vestuário, entre outros (IBGE, 2007).

O ajuste do PIB para preços constantes de 2005 foi feito multiplicando-se o índice de preços cumulativamente a cada ano, pelo valor do PIB a preços correntes do ano equivalente, a partir de 2005, o ano mais recente em que o dado estava disponível na literatura.

\section{Projeção dos cenários}

Os dados históricos da produção de RSU - a variável dependente ou resposta -, foram correlacionados linearmente através de regressão múltipla (DYSON; CHANG, 2005; NIESSEN; ALSOBROOK, 1972; GROSSMAN; HUDSON; MARK, 1974) com os dados históricos de População e PIB total de Curitiba - variáveis explicativas ou independentes - no intuito de utilizar as variáveis explicativas para prever ou explicar o comportamento da geração de RSU no futuro. Para Sokka, Antikainen e Kauppi (2007), Adhikari, Barringt e Martinez (2006) e Daskalopoulos, Badr e Probert (1998) os parâmetros-chave que melhor explicam a geração de resíduos sólidos urbanos são população e padrão de vida, sendo PIB o indicador para o padrão de vida da população

O programa Kyplot 2.0 (YOSHIOKA, 2002) foi utilizado para análise estatística dos dados históricos referentes à produção de resíduos sólidos urbanos em Curitiba entre 1995 e 2007: RSU (Y), população (X1) e PIB Total (X2). A análise dos dados pelo programa sugeriu a existência de multicolineariedade entre as variáveis e, dessa forma, foi obtida a Equação 1, que calcula a variável RSU em função da População e do PIB, com coeficiente de correlação múltipla $\mathrm{R}^{2}=0,84$

$\mathrm{RSU}=1,5657 * \mathrm{PIB}-3,6861 * \mathrm{POP}+5,5416$

Equação 1

Para obter os valores de RSU nos anos futuros, foi utilizada a Equação 1 alimentada com dados anuais de população e PIB total projetados até 2020. Para os dados populacionais futuros, foi utilizada a projeção populacional do IPARDES (2008), e para o PIB futuro, assumiu-se a tendência linear da evolução histórica, uma vez que a evolução dessa variável ao longo dos anos apresentou tendência linear com alto grau de correlação $\left(R^{2}=0,95\right)$.

\section{Análise comparativa dos cenários futuros alternativos}

Ao todo, foram construídos sete cenários alternativos da produção de resíduos sólidos urbanos em Curitiba compreendendo o período de 2008 a 2020. Para a análise dos resultados, foram utilizados alguns indicadores gerais disponíveis na literatura, como uma forma simplificada de valorar impactos ambientais e econômicos de cada cenário no futuro (Tabela 1). Para analisar os efeitos dos resíduos orgânicos em cada cenário, foi utilizada a Equação 2, empregada por Adhikari, Barringt e Martinez (2006) para estimar a produção de metano a partir de resíduos orgânicos:

$\left(\mathrm{CH}_{4} \mathrm{P}\right)=\alpha *(\% \mathrm{DW} / 100) *(\mathrm{RSU})$

Equação 2

Onde:

$\mathrm{CH}_{4} \mathrm{P}$ : produção de metano $\left(\mathrm{t}^{*}\right.$ ano $\left.{ }^{-1}\right)$;

$\alpha$ : potencial de produção de metano $\left(0,204 \mathrm{~kg}\right.$ de metano. $\mathrm{kg}^{-1} \mathrm{de}$ peso seco, segundo Wang et al, 1997);

DW: peso seco em média dos resíduos orgânicos (30\% segundo Peavy, Rowe e Tchobanoglous, 1985);

RSU: produção de resíduos sólidos urbanos (ADHIKARI; BARRINGT; MARTINEZ, 2006). 
Tabela 1 - Indicadores utilizados para estimativa dos impactos da geração de RSU em Curitiba

\begin{tabular}{lc}
\hline Indicadores e fontes & \multicolumn{1}{c}{ Valor } \\
\hline Densidade dos RSU (MONTEIRO, 2006; USEPA, 2005) & $600 \mathrm{~kg} \cdot \mathrm{m}^{-3}$ \\
\hline Densidade dos Recicláveis (FARIAS, 2002) & $300 \mathrm{~kg} \cdot \mathrm{m}^{-3}$ \\
\hline $\begin{array}{l}\text { Densidade dos Resíduos Orgânicos (QUARESMA, 1998) } \\
\text { Custo médio de Coleta em Curitiba (MINISTÉRIO DAS }\end{array}$ & $\mathrm{R} \$ 48,48 \cdot \mathrm{t}^{-3}$ \\
$\begin{array}{l}\text { CIDADES, 2003) } \\
\text { Custo do Aterramento (PAES et al, 2007) }\end{array}$ & $\mathrm{R} \$ 23,00 . \mathrm{t}^{-1}$
\end{tabular}

\section{Resultados e Discussão}

\section{Caracterização da geração de RSU em Curitiba}

Em Curitiba, a produção total de resíduos sólidos urbanos tem atingido valores cada vez mais altos ao longo dos anos. De 1990 a 2007, a geração anual mais que dobrou: em 1990, a produção total foi de 188.629 toneladas e, em 2007, foi a maior do período, ultrapassando 400 mil toneladas. Nesse mesmo intervalo, a taxa de geração per capita dos resíduos sólidos urbanos também aumentou, passando de 0,402 kg.hab-1. dia-1 em 1990 para 0,613 kg.hab-1.dia-1 (Figura 1).

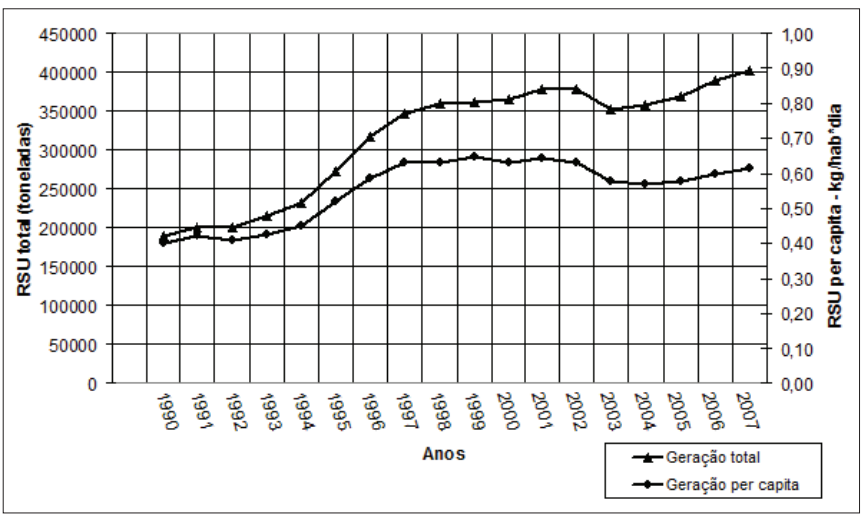

Figura 1 - Evolução da geração total e per capita de Resíduos Sólidos Urbanos de Curitiba entre 1990 e 2007

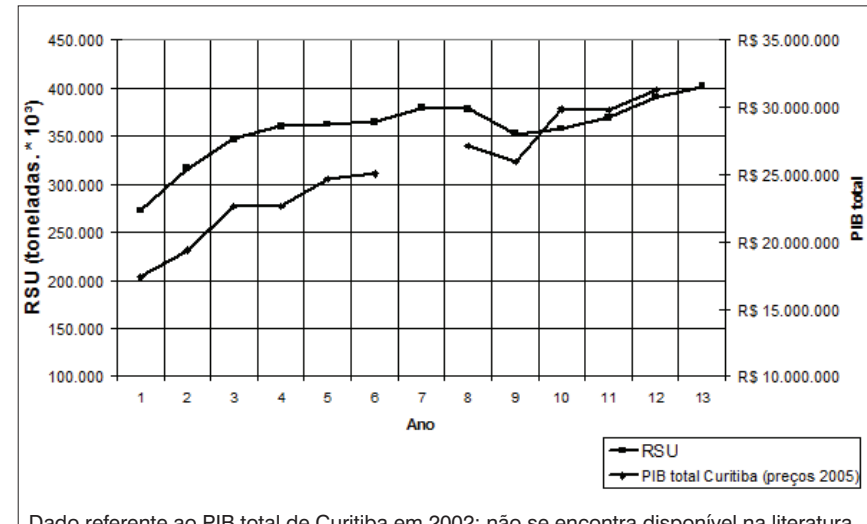

Figura 2 - Evolução da geração total de resíduos sólidos urbanos e PIB total de Curitiba
Segundo a média dos últimos cinco anos, são coletadas anualmente cerca de 374,6 mil toneladas de resíduos sólidos urbanos em Curitiba. Isso equivale à geração diária de pouco mais de 1.027 toneladas. A geração per capita é de aproximadamente $0,59 \mathrm{~kg} \cdot \mathrm{hab}^{-1} \cdot \mathrm{dia}^{-1}$, no mesmo período (PAES et al, 2007; IBGE, 2007).

A geração per capita de RSU em Curitiba é consideravelmente baixa em relação à média brasileira $\left(0,95 \mathrm{~kg} \cdot \mathrm{hab}^{-1} \cdot \mathrm{dia}^{-1}\right)$ e tem quase metade do valor que se esperaria para uma cidade desse porte populacional (1,16 kg.hab . dia $\left.^{-1}\right)$, segundo IBGE (2002). O crescimento mais acelerado ocorreu logo após 1994, ano em que foi criado o Plano Real e houve declínio considerável dos índices inflacionários, gerando um aumento no consumo. Com exceção dos anos 2002 e 2003, houve crescimento na geração total de resíduos sólidos entre 1990 e 2007.

Segundo Sokka, Antikainen e Kauppi (2007) e Medina (1997), muito do crescimento na produção de resíduos sólidos urbanos pode ser atribuído ao crescimento do PIB. No ano de 2003, houve um notável declínio da produção de RSU. Em comparação à evolução do PIB da cidade, é possível notar que 2003 não foi um bom ano para a economia da cidade, e houve um decréscimo considerável do PIB total em relação ao ano anterior, o que não ocorreu em nenhum dos outros anos verificados. Analisando-se a evolução do índice de preços IPCA (IBGE, 2007) ao longo da última década, constatou-se que a inflação em Curitiba atingiu seu pico em 2002, o que significa que, no início de 2003, os produtos e serviços estavam bem mais caros em relação ao ano anterior. Essas evidências podem sugerir que a redução na produção de resíduos sólidos urbanos em Curitiba naquele ano pode ter sido causada, dentre outros fatores, pelo decréscimo do poder de compra da população (Figura 2).

Segundo Parizeau, Maclaren e Chanty (2006), no gerenciamento de resíduos sólidos urbanos, é necessário conhecer as características dos resíduos gerados pela população. O conhecimento da composição destes resíduos é fator primordial no planejamento da gestão, consideração de investimentos em coleta, tratamento, disposição final e desenvolvimento de programas de gestão. A composição dos RSU em Curitiba tem sido pouco estudada, e apenas recentemente um estudo de caracterização gravimétrica com bases científicas foi realizado (TAVARES, 2007).

A composição dos resíduos sólidos urbanos de Curitiba (Figura 3) mostra que a porcentagem de resíduos recicláveis em relação ao total (potencial de reciclagem) é de 40,5\% (PAES et al, 2007), sendo superior aos 30,8\% de São Paulo e 38,4\% do Rio de Janeiro (SANTOS, 2004). Em relação aos resíduos orgânicos, a porcentagem nos resíduos urbanos de Curitiba (47,9\%) é inferior aos valores dessas cidades (64,4 e 51,6\%, respectivamente).

Em Curitiba não é feita a compostagem de resíduos orgânicos como parte do processo de gerenciamento dos resíduos sólidos urbanos (PAES et al, 2007). Por outro lado, a cidade conta com programa de coleta seletiva e reciclagem desde 1990, tendo sido a cidade pioneira no Brasil a implantar programas desse tipo (PAES et al, 2007). 
Segundo CEMPRE (2000), a cidade de Curitiba reciclava, em 1999, aproximadamente $15 \%$ de seus resíduos (considerando a coleta seletiva municipal e o trabalho dos catadores), um índice alto mesmo comparado a países desenvolvidos (10\% em Barcelona, Espanha; 15\% na Bavária, Alemanha).

A evolução da quantidade de material reciclável coletado pelo programa de coleta seletiva em Curitiba mostra que, desde a sua criação até 1999, a quantidade de materiais coletados foi crescente ao longo dos anos. No entanto, de 1999 a 2005, houve um considerável declínio da quantidade de materiais coletados (PAES et al, 2007). De acordo com Tavares (2007) e Paes et al (2007), alguns dos motivos para o declínio na coleta seletiva em Curitiba podem ter sido: o aumento do número de carrinheiros; os contingentes populacionais migratórios provenientes de outras cidades e Estados para Curitiba, atraídos pela industrialização do município, desacostumados com a separação dos resíduos recicláveis; baixos investimentos no programa da coleta seletiva e, consequentemente, na educação ambiental da população em relação à separação dos recicláveis. A partir de 2006, com o lançamento da nova campanha de educação para a separação do "lixo que não é lixo" pela Prefeitura Municipal, a quantidade de materiais coletados voltou a aumentar.

Em relação à destinação dos resíduos sólidos urbanos, ainda é utilizado o Aterro da Caximba, um aterro sanitário que recebe os resíduos sólidos da região metropolitana de Curitiba há 17 anos e que teve detectada a sua capacidade esgotada antes de uma nova solução ser encontrada. Para o projeto do aterro, foi adotada uma produção per capita média de lixo de $0.55 \mathrm{~kg}$.hab ${ }^{-1}$. dia ${ }^{-1}$ (PAES et al, 2007).

\section{Projeção da produção de resíduos sólidos urbanos em Curitiba}

A Figura 4 mostra a projeção linear da geração de resíduos sólidos urbanos em Curitiba de 2008 a 2020, com base no comportamento histórico dos dados socioeconômicos levantados para o período anterior a 2008.

Como exposto na metodologia, a equação utilizada para a projeção dos RSU em Curitiba leva em consideração dois fatores - População e PIB - cobrindo alguns dos principais fatores socioeconômicos que podem guiar a produção de resíduos urbanos. Mas existem outros fatores que podem influenciar a geração de resíduos. O trabalho de Sokka, Antikainen e Kauppi (2007), por exemplo, utiliza um indicador para o fator Tecnologia, considerando-se que o desenvolvimento tecnológico também exerce influência na geração de resíduos sólidos urbanos, ou seja, é uma variável indicativa da eficiência de produção.

Devido às considerações aqui mencionadas, os resultados da projeção de resíduos urbanos em Curitiba devem ser tratados com cuidado. Eles são indicativos de uma tendência histórica que mostra como os fatores considerados podem afetar a geração de resíduos no futuro, relacionando a geração de RSU e população e PIB. Para estimativas

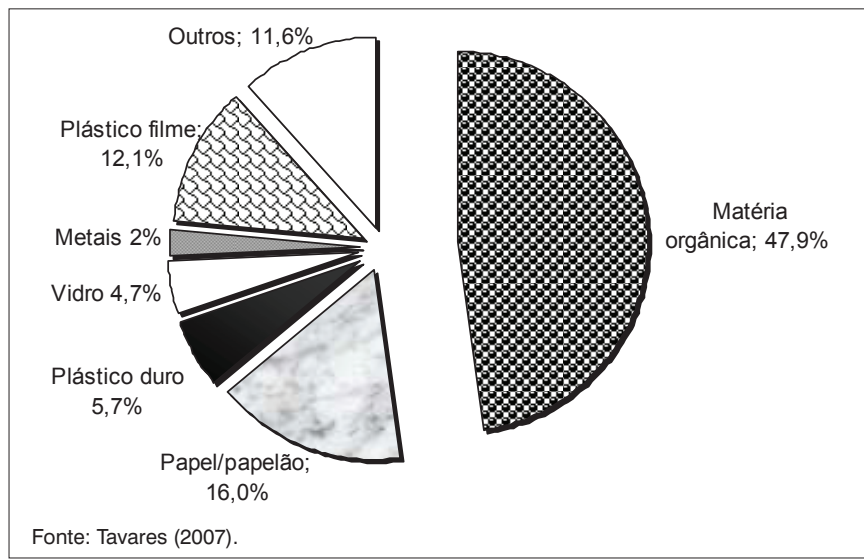

Figura 3 - Composição relativa dos resíduos urbanos de Curitiba

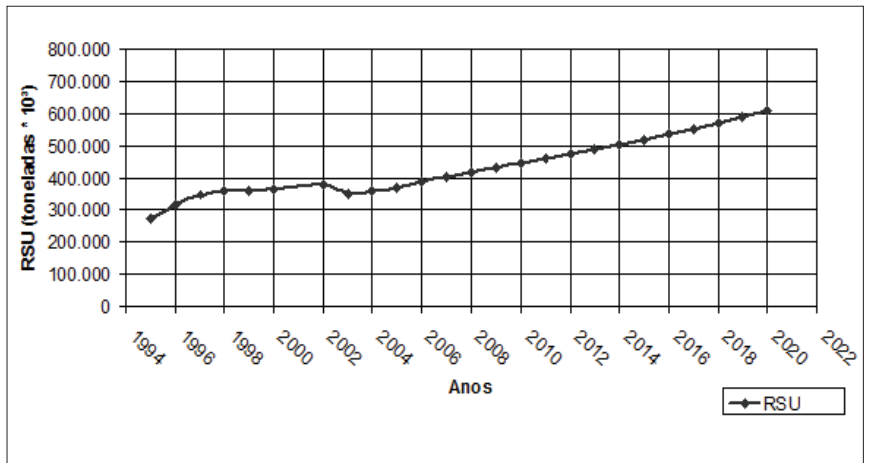

Figura 4 - Projeção da geração de resíduos sólidos urbanos em Curitiba -2008 a 2020

mais precisas, modelos mais complexos como os utilizados por Dyson e Chang (2005) e Bruvoll e Ibenholt (1997) poderiam ser aplicados.

\section{Cenários alternativos da produção de resíduos sólidos urbanos em Curitiba}

A Tabela 2 traz um resumo dos cenários construídos, com as estimativas da influência de cada um, para fins comparativos. Os cenários foram construídos sob algumas hipóteses particulares, sendo, portanto, apenas exemplos de aplicação do método. As hipóteses poderiam ser também cumulativas, gerando impactos cumulativos, criando outros cenários. Na verdade, a possibilidade de construção de cenários é ilimitada e a vantagem da técnica de cenários é a possibilidade de visualização e comparação entre cada cenário.

O cenário 1 foi construído a partir da tendência histórica da geração. É o cenário que Adhikari et al (2006) chama de cenário "do nothing". Esse cenário seria o mais provavelmente verdadeiro caso as estratégias e políticas de gerenciamento fossem mantidas no futuro. Além disso, assume-se a hipótese de que a composição dos resíduos se manteria inalterada em relação ao status quo (TAVARES, 2007). O cenário 1 foi utilizado como referência para a construção dos outros cenários, sendo, portanto, considerado o cenário da tendência histórica. 
Tabela 2 - Tabela comparativa dos resultados dos cenários alternativos

\begin{tabular}{lccccc} 
Cenários (Metas) & $\begin{array}{c}\text { Geração anual de } \\
\text { RSU em 2020 }\end{array}$ & $\begin{array}{c}\text { Quantidade em } \\
\text { peso }(\mathrm{t}) \text { 2008- } \\
2020\end{array}$ & $\begin{array}{c}\text { Quantidade em } \\
\text { volume }\left(\mathrm{m}^{3}\right) \\
2008-2020\end{array}$ & $\begin{array}{c}\text { Custo para geren- } \\
\text { ciamento }\end{array}$ & $\begin{array}{c}\text { Geração de } \\
\text { metano }(\mathrm{t}) \\
2008-2020\end{array}$ \\
\hline Cenário 1 (do nothing) & 609.003 & 6.603 .574 & 11.005 .574 & $\mathrm{R} \$ 29.760 .367$ & 193.582 \\
Cenário 2 (-5\% geração total) & 578.553 & 6.273 .395 & 10.455 .659 & $\mathrm{R} \$ 28.272 .348$ & 183.903 \\
Cenário 3 (+5\% geração total) & 639.453 & 6.933 .753 & 11.556 .255 & $\mathrm{R} \$ 31.248 .385$ & 203.262 \\
Cenário 4 (-20\% recicláveis) & 559.674 & 6.068 .684 & 10.114 .091 & $\mathrm{R} \$ 27.349 .777$ & 193.582 \\
Cenário 5 (-10\% orgânicos) & 578.735 & 6.275 .376 & 10.458 .578 & $\mathrm{R} \$ 28.281 .276$ & 183.961 \\
Cenário 6 (-20\% recicláveis e -10\% orgânicos) & 529.406 & 5.740 .487 & 10.142 .487 & $\mathrm{R} \$ 25.870 .686$ & 168.281 \\
\hline
\end{tabular}

Os cenários 2 e 3 foram construídos a partir do aspecto de interesse da geração total de resíduos sólidos. Nos cenário 2 e 3, supõe-se que haveria, respectivamente, redução e aumento de 5\% na geração total de resíduos sólidos urbanos na cidade. Essas hipóteses poderiam ocorrer, por exemplo, se houvessem a mudanças nas práticas de gestão, como a intensificação de programas de minimização da geração, ou nos hábitos de consumo da população, com aumento da quantidade de resíduos gerados por cada habitante. Em relação ao cenário 1, as diferenças - que se traduzem em economia para o cenário 2 e gastos adicionais para o cenário 3 - seriam: 330.179 toneladas geradas, $550.298 \mathrm{~m}^{3}$ de volume no aterro, e R\$ 1.488.018,00 para o gerenciamento dos resíduos.

O cenário 4 foi construído a partir do aspecto de interesse da separação dos materiais recicláveis presentes nos resíduos urbanos. Considerou-se uma hipótese de redução na quantidade de recicláveis destinados ao aterro, o que poderia ocorrer no futuro caso programas de reciclagem fossem intensificados e desenvolvidos, e esforços do gerenciamento fossem direcionados para o aspecto da separação e reciclagem. Em comparação ao cenário "do nothing", até 2020 as diferenças seriam de: 534.890 toneladas geradas, $891.483 \mathrm{~m}^{3}$ de volume no aterro e R\$2.410.560,00 para o gerenciamento desses resíduos. Outro aspecto que poderia ser analisado diz respeito ao valor de mercado desses materiais; se pudessem de fato ser comercializados, gerariam ainda uma potencial receita de $\mathrm{R} \$ 684.803,00$, de acordo com o valor atual de mercado de cada material (HOLZMANN, 2008)․․

O cenário 5 foi construído considerando-se a possibilidade de compostagem dos resíduos orgânicos presentes nos resíduos urbanos que normalmente são enviados ao aterro sanitário. Considerando, por exemplo, a possível existência de um sistema de compostagem na cidade, com o qual poderia haver uma redução de 10\% na quantidade de resíduos orgânicos enviadas ao aterro em comparação ao cenário "do nothing", até 2020 haveria uma redução de 328.198 toneladas geradas, $546.996 \mathrm{~m}^{3}$ de volume no aterro e $\mathrm{R} \$ 1.479 .090,00$ para o gerenciamento. Um dos impactos de maior interesse na geração de resíduos orgânicos é o biogás (principalmente $\mathrm{CH}_{4}$ ) gerado pela decomposição da matéria orgânica. Utilizando a Equação 2, para estimar a produção de metano a partir de quantidades em peso de resíduos orgânicos, constata-se que no cenário 5 haveria geração de 183.961 ton. Isso significa que, em relação ao cenário 1 , um total de 9.622 ton. de metano originado pela decomposição dos resíduos orgânicos seria evitada (cenário 5).

O cenário 6 ilustra uma possível situação em que, através de estratégias de intensificação da reciclagem e compostagem, seria possível reduzir as quantidades de resíduos orgânicos e de materiais recicláveis enviadas ao aterro sanitário. Em relação ao cenário "do nothing", isto representaria até 2020 uma redução de: 863.087 toneladas geradas, $1.438 .478,61$ de volume no aterro, $\mathrm{R} \$ 3.889 .679,00$ para o gerenciamento. Em relação à produção de metano, haveria geração de 168.281 toneladas. Isso significa que, em relação ao cenário 1, a emissão e 25.301 toneladas de metano originado pela decomposição dos resíduos orgânicos seria evitada.

O grande percentual de geração de recicláveis observado nos resíduos sólidos urbanos de Curitiba dá subsídios e validade ao argumento de que medidas poderiam ser tomadas para diminuir a quantidade de materiais recicláveis encaminhados ao aterro sanitário de Caximba, aumentando a vida útil do mesmo. A coleta seletiva de materiais pode ser substancialmente melhorada a partir da adoção de três frentes: (a) alteração das técnicas de intervenção, apostando na sensibilização da população priorizando-se a não-geração e minimização, (b) abrangência do setor comercial e industrial nos programas de coleta seletiva, (c) maiores investimentos em sistemas de reciclagem, visando à recuperação destes materiais (PUNA; BAPTISTA, 2008). Segundo GonçalvesDias (2006), o setor público pode atuar no sentido de implantar boas práticas, como a coleta seletiva, além de incentivar a organização de cooperativas de carrinheiros e a promoção de uma legislação que incentive a reciclagem. Este incentivo pode vir da adoção de mecanismos fiscais, por exemplo, concedendo benefícios às indústrias recicladoras, de imposições legais e da aplicação de mecanismos econômicos, que devem ser flexíveis e orientados para o mercado (PUNA; BAPTISTA, 2008). Os mesmos autores ainda consideram necessário o estudo de aplicação de taxas variáveis ou tarifas proporcionais à quantidade de resíduos produzidos por cada família, para seu encaminhamento ao Aterro. Estas taxas devem ser calculadas de forma a cobrir parcial ou totalmente os custos de coleta, transporte e processamento, funcionando como um incentivo à redução da geração de resíduos dentro das casas. Walsh (2002) ainda recomenda o incentivo ao desenvolvimento de um mercado de separação, coleta, reciclagem e comercialização 
dos resíduos sólidos urbanos. Sendo o desenvolvimento de tecnologias que permitam materiais mais fáceis de reciclagem, inofensivos e inertes (GONÇALVES-DIAS, 2006) importante para o desenvolvimento deste mercado. Por outro lado, a eficácia da reciclagem depende da consciência ambiental da população e de uma eficiente segregação dos resíduos recicláveis na fonte geradora. Para isso, as campanhas e programas de educação da população devem ser intensificados e mais bem planejados. Deve-se levar ao grande público o conhecimento sobre reciclagem dos materiais, instruindo-o sobre como proceder ao correto descarte dos resíduos (GONÇALVES-DIAS, 2006). Quanto aos resíduos orgânicos, diante da grande porcentagem em relação ao total dos resíduos sólidos urbanos gerados em Curitiba, e considerando-se o seu potencial de tratabilidade via compostagem, eles poderiam ser destinados e tratados em uma usina de compostagem. Além da economia de espaço no aterro, que contribuiria para o aumento da sua vida útil, haveria diminuição de emissões gasosas prejudiciais ao meio ambiente. Segundo o IBGE (2002), no Brasil existiam, em 2000, 260 usinas de compostagem de resíduos orgânicos, sendo que 12 estão localizadas no Paraná - nenhuma delas em Curitiba.

Os resultados dos cenários desse estudo mostram a complexidade do fenômeno urbano de geração de resíduos, trazendo diferentes perspectivas para a geração futura de resíduos sólidos urbanos em Curitiba. Os gestores responsáveis pelo planejamento devem estar cientes de todas as variáveis e incertezas (SOKKA; ANTIKAINEN; KAUPPI, 2007), sendo necessária a capacitação destes gestores para a mudança dos cenários estabelecidos. O hábito das pessoas, no que diz respeito ao tratamento dos resíduos gerados em suas residências, é influenciado, entre outras coisas, pela percepção que têm do serviço de coleta de sua cidade. Suas percepções desse serviço público, se realizado de forma descompromissada, estimulam atitudes despreocupadas (MUCELIN; BELLINI, 2008). Por isto deve-se atentar ao fato da necessidade de investimento, por parte do Poder Público, em propaganda e educação ambiental, mobilizando a sociedade para o correto gerenciamento de seus resíduos. Leis regulamentando a atividade, bem como o estabelecimento de padrões e procedimentos, também devem ser considerados pelo Poder Público. Neste sentido, consórcios de municípios limítrofes permitem a otimização de esforços. Porém, outras alternativas também devem ser levadas examinadas para a formulação de Políticas Públicas sobre resíduos sólidos. A moderna abordagem do gerenciamento de resíduos prevê uma hierarquia onde deve ser dada preferência à não geração de resíduos, seguindo-se, dentro de possibilidades econômicas e tecnológicas, a minimização, o reaproveitamento (reciclagem, recuperação e reuso) e somente após isto, o tratamento e a disposição final (VALLE, 2000). A cogeração de energia a partir da incineração dos resíduos (waste-to-energy) também pode ser uma alternativa a ser considerada pelos tomadores de decisão.

Os cenários servem como instrumentos para o norteamento de políticas e estratégias de gerenciamento de resíduos sólidos urbanos e, sendo assim, para se minimizarem erros (na consideração de investimentos, por exemplo), o aperfeiçoamento das estatísticas e dos dados referentes à produção de resíduos deve ser contínuo.

\section{Conclusões}

Pelos resultados desse estudo, estima-se que a geração de resíduos sólidos urbanos em Curitiba será crescente até 2020, ultrapassando 600 mil toneladas anuais. Porém, as quantidades de resíduo geradas e as suas consequências podem ser reduzidos por meio de ações e estratégias de gerenciamento.

A possibilidade de construção de cenários futuros hipotéticos da produção de RSU em Curitiba, nos quais diferentes estratégias e metas foram consideradas, trouxe resultados sob a forma de estimativas de consequências, permitindo que os efeitos de cada cenário pudessem ser visualizados e comparados. Dessa forma, é possível planejar a longo prazo a gestão de resíduos sólidos na cidade, definindo-se metas e as melhores formas de alcançá-las por meio da minimização, reciclagem e compostagem.

Existe a necessidade de mais estudos sobre o tema, e como sugestão para outros estudos, poderiam ser feitos em um universo maior de cidades da região metropolitana de Curitiba. Outra possibilidade seria aplicar o método de estudo a outros tipos de resíduos, como os resíduos de serviços da saúde ou os resíduos industriais. Outras metodologias para projeção da geração dos resíduos sólidos podem ser desenvolvidas e aplicadas na construção de cenários futuros, considerando-se um número maior de variáveis críticas, como fatores tecnológicos ou fatores culturais, por exemplo.

\section{Referências}

ADHIKARI, K. B.; BARRINGT, S.; MARTINEZ, J. Predicted growth of world food waste and methane production. Waste Management and Research, v. 24, n. 5, p. 421-433, 2006.

AMORIN, V.P. Resíduos sólidos urbanos: o problema e a solução. Brasília, DF: Roteiro Editorial Ltda, 1996.
BRUVOLL, A.; IBENHOLT, K. Future waste generation forecasts on the basis of a macroeconomic model. Resources, Conservation and Recycling, v. 19, n. 3, p. 137-149, 1997

BUARQUE, C.S. Metodologia e técnicas de construção de cenários globais e regionais. Brasília, DF: IPEA, 2003. 
CARIDADE, A.V.S.; WRIGHT, J.T.C.; SPERS, R.G. Técnicas de cenários, aplicação prática de prospecção de cenários: Profuturo e o estudo prospectivo sobre os consumidores do futuro. In: SEMINÁRIOS EM ADMINISTRAÇÃO VIII, São Paulo, 2005.

COMPROMISSO EMPRESARIAL PARA RECICLAGEM (CEMPRE). LiXO municipal: manual de gerenciamento integrado. $2^{a}$ Ed. CEMPRE: São Paulo, 2000.

DASKALOPOULOS, E.; BADR, O.; PROBERT, S.D. Municipal solid waste: a prediction methodology for the generation rate and composition in the European Union and the United States of America. Resources, Conservation and Recycling, v. 24, n. 1, p. 155-166, 1998.

DYSON, B.; CHANG, N.B. Forecasting municipal solid waste generation in a fast growing region with system dynamics modeling. Waste Management, v. 25, n. 7, p. 669-679, 2005.

GONÇALVES-DIAS, S.L.F. Há vida após a morte: um (re)pensar estratégico para o fim da vida das embalagens. Gestão \& Produção, v. 13 , n. 3, p. 463-474, 2006

GROSSMAN, D; HUDSON, J.F.; MARKS, D.H.; ASCE, M. Waste generation models for solid waste collection, Journal of the Environmental Engineering Division, v.100, p.1219-1230, 1974.

INSTITUTO BRASILEIRO DE GEOGRAFIA E ESTATÍSTICA (IBGE). [Online]. Disponível em: <www.ibge.gov.br> Acesso em 3 nov. 2009.

Pesquisa Nacional de Saneamento Básico, PNSB 2000. Rio de Janeiro: IBGE, 2002.

INSTITUTO PARANAENSE DE DESENVOLVIMENTO E ECONÔMICO E SOCIAL (IPARDES). Projeção da população de Curitiba BDEWEB. [Online]. Disponível em: <www.ipardes.gov.br/imp/index.php> Acesso em: 23 ago. 2008

INSTITUTO DE PESQUISA DE CURITIBA (IPPUC). [On-line]. Disponível em: <www.ippuc.pr.gov.br> Acesso em: 3 nov. 2009.

MEDINA, M. Globalization, development, and municipal solid waste management in third world cities. El Colegio de la frontera Norte, Tijuana, México. 1997. Disponível em: www.gdnet.org/ pdf/2002AwardMedalWinners/outstandingresearchdevelopment/ martin_medina_martinez_paper.pdf> Acesso em: 3 nov. 2009.

MINISTÉRIO DAS CIDADES. Avaliação regional dos serviços de manejo de resíduos sólidos municipais. Informe analítico da situação da gestão municipal de resíduos sólidos no Brasil 2002. Secretaria Nacional de Saneamento Ambiental Programa de Modernização do Setor de Saneamento: São Paulo, 2003.

MONTEIRO, E.D.V. Estudo do comportamento de RSU em uma célula experimental e suas correlações com aspectos microbiológicos, físicos e químicos. Engenharia Sanitária e Ambiental, v. 11, n. 3, p. 223-230, 2006.

MUCELIN, C.A.; BELLINI, M. Lixo e impactos ambientais perceptíveis no ecossistema urbano. Sociedade \& Natureza, v. 20, n. 1, p. 111-124, 2008.
NIESSEN, W.R.; ALSOBROOK, A.F. Municipal and industrial refuse: composition and rates, Proceedings of the 1970 National Incinerator Conference, American Society of Mechanical Engineers, New York, USA (1972), pp. 319-337.

PAES, N.X. et al. Gerenciamento dos Resíduos Sólidos Urbanos no Município de Curitiba. Secretaria Municipal do Meio Ambiente/ Departamento de Limpeza Pública: Curitiba, 2007.

PARIZEAU, K.; MACLAREN, V.; CHANTY, L. Waste characterization as an element of waste management planning. Lessons learned from a study in Siem Reap, Cambodia. Resources, Conservation and Recycling, v. 49, n. 2, p. 110-128, 2006.

PEAVEY, H.S.; ROWE, D.R.; TCHOBANOGLOUS, G. Environmental engineering. McGraw Hill: New York, 1985

PUNA, J.F.B.; BAPTISTA, B.S. A gestão integrada de residuos sólidos urbanos - persperctiva ambiental e economico-energética. Química Nova, v. 31, n. 3, p. 645-654, 2008.

QUARESMA, J.B. Proposta para tratamento dos resíduos sólidos na cidade de Monte Alegre. Ministério de Minas e Energia e Governo do Estado do Pará: Monte Alegre, 1998.

SANTOS, L.C. A questão dos resíduos sólidos urbanos: uma abordagem socioambiental com ênfase no Município de Ribeirão Preto (SP). 120 f. Dissertação (Mestrado em Engenharia Civil) - Instituto de Geociências e Ciências Exatas, Rio Claro, 2004

SOKKA, L.; ANTIKAINEN, R.; KAUPPI, P.E. Municipal solid waste production and composition in Finland - Changes in the period 19602002 and prospects until 2020. Resources, Conservations and Recycling v. 50, n. 4 , p. $475-488,2007$.

TAVARES, R.C. Composição gravimétrica: uma ferramenta de planejamento e gerenciamento do resíduo urbano de Curitiba e região metropolitana. 114f. Dissertação (Mestrado) - Instituto de Engenharia do Paraná, Instituto de Tecnologia para o Desenvolvimento, Curitiba, 2007.

UNITED STATES ENVIRONMENTAL PROTECTION AGENCY (USEPA). Municipal solid waste in the United States: 2005 facts and figures, executive summary. 2005. Disponível em: <http://www.epa.gov>. Acesso em: 25 nov. 2006.

VALLE, C.E. Como se preparar para as normas ISO 14000: Qualidade ambiental. 3 ed. Pioneira: São Paulo, 2000

WALSH, D.C. Urban residential refuse composition and generation rates for $20^{\text {th }}$ century. Environment Science Technology, v. 36, n. 22, p. 49364942, 2002.

WANG, Y.S. et al. Methane potential of food waste and anaerobic toxicity of leachate production produced during food waste decomposition. Waste Management and Research, v. 15, n. 2, p. 149-167, 1997.

YOSHIOKA, K. Kyplot: statistical tool for data analysis and visualization. Department of Biochemistry and Biophysics, Graduate School of Allied Health Sciences, Tokyo Medical and Dental University. Tokyo, 2002. 